Pacific Journal of Mathematics

INSULAR PERTURBATION OF A TIME-DEPENDENT 


\section{SINGULAR PERTURBATION OF A TIME-DEPENDENT CAUCHY PROBLEM IN A HILBERT SPACE}

\section{E. Bobisud and James Calvert}

Let $A$ be a self-adjoint operator, not necessarily bounded, in the Hilbert space $H$, with resolution of the identity $E_{2}$. Define $h(t, A)=\int_{-\infty}^{\infty} h(t, \lambda) d E_{\lambda}$. It is shown that as $\varepsilon \rightarrow 0+$ the solution of the abstract problem $\varepsilon U_{\varepsilon}^{\prime \prime}+b U_{\varepsilon}^{\prime}+h(t, A) U_{\varepsilon}=$ $0, U_{\varepsilon}(0)=x_{0}, U_{\varepsilon}^{\prime}(0)=x_{1}$ tends in the norm of $H$ to the solution of $b U_{0}^{\prime}+h(t, A) U_{0}=0, U_{0}(0)=x_{0}$ for data $x_{0}, x_{1}$ in a dense subset of $H$.

Let $A$ be a (possibly unbounded) self-adjoint operator in a Hilbert space $H$, and let $E_{2}$, be the resolution of the identity for $A$, so that

$$
A x=\int_{-\infty}^{\infty} \lambda d E_{\lambda} x
$$

for $x \in D(A) \subset H$. Let $h(t, \lambda)$ defined on $[0, \infty) \times(-\infty, \infty)$ be a Borel measurable function of $\lambda$ for fixed $t$ and a continuous function of $t$ for fixed real $\lambda$. Then an operator $h(t, A)$ can be defined by

$$
h(t, A) x=\int_{-\infty}^{\infty} h(t, \lambda) d E_{2} x
$$

for $x \in D(h(t, A))$, where

$$
D(h(t, A))=\left\{x \in H: \int_{-\infty}^{\infty}|h(t, \lambda)|^{2} d\left\|E_{\lambda} x\right\|^{2}<\infty\right\} .
$$

We shall be concerned with the behavior as $\varepsilon \rightarrow 0+$ of the solution of the problem

$$
\varepsilon U_{\varepsilon}^{\prime \prime}+b U_{\varepsilon}^{\prime}+h(t, A) U_{\varepsilon}=0, U_{\varepsilon}(0)=x_{0}, U_{\varepsilon}^{\prime}(0)=x_{1},
$$

where $b$ is a positive constant. It seems reasonable to expect that $U_{\varepsilon} \rightarrow U_{0}$ as $\varepsilon \rightarrow 0$, where $U_{0}$ solves the problem

$$
b U_{0}^{\prime}+h(t, A) U_{0}=0, U_{0}(0)=x_{0} .
$$

We prove this convergence, as well as $U_{\varepsilon}^{\prime} \rightarrow U_{0}^{\prime}$, in the norm of $H$ for data $x_{0}, x_{1}$ restricted to a certain dense subset of $H$.

Several abstract singular perturbation problems of this nature have been considered before. Kisyński [5] considered the case $h(t, A) \equiv A$ where $A$ is positive as well as self-adjoint; in addition, he considered the inhomogeneous problem. Smoller [9, 10], Latil [6], Friedman [4] and the authors [1] have extended his results to higher- 
order equations and have removed the restriction that $A$ be positive. The use of the resolution of the identity for $A$ and estimates for the special case $H=L^{2}(-\infty, \infty), A=$ a real parameter $\lambda$ is central to all these treatments, as well as to the present study. Singular perturbations in Banach spaces have been studied by Bobisud and Hersh [2], Sova [11], and Schoene [8].

Time-dependent equations of the form

$$
\varepsilon p(t) U_{\varepsilon}^{\prime \prime}+q(t) U_{\varepsilon}^{\prime}+A U_{\varepsilon}=0
$$

and higher-order generalizations have been considered by Friedman [4]. The only previous study of a nonfactorable time-variable operator $h(t, A)$ which is known to the authors is that of Nur [7], who considers the case $h(t, \lambda)=e^{\lambda t}$, so $h(t, A)$ is a semigroup with generator $A$. The result of Nur is contained in the theorems to follow.

As mentioned above, we begin by examining in part 1 the special case $H=L^{2}(-\infty, \infty)$ and show that $u_{\varepsilon}(t, \lambda) \rightarrow u_{0}(t, \lambda)$, where

$$
\begin{gathered}
\varepsilon u_{\varepsilon}^{\prime \prime}+b u_{\varepsilon}^{\prime}+h(t, \lambda) u_{\varepsilon}=0, u_{\varepsilon}(0, \lambda)=x_{0}, u_{\varepsilon}^{\prime}(0, \lambda)=x_{1}, \\
b u_{0}^{\prime}+h(t, \lambda) u_{0}=0, \quad u_{0}(0, \lambda)=x_{0} .
\end{gathered}
$$

We also establish for this case certain estimates to be used in treating the Hilbert space problem in part 2.

1. The problem on the real line. Since the problems (3), (4) are linear, we may write

$$
\begin{aligned}
& u_{\varepsilon}(t, \lambda)=p_{\varepsilon}(t, \lambda) x_{0}+q_{\varepsilon}(t, \lambda) x_{1}, \\
& u_{0}(t, \lambda)=p_{0}(t, \lambda) x_{0}
\end{aligned}
$$

for certain functions $p_{\varepsilon}, q_{\varepsilon}, p_{0}$. Regarding a solution of (3) for fixed $\varepsilon$ as a solution of the equation $\varepsilon u_{\varepsilon}^{\prime \prime}+b u_{\varepsilon}^{\prime}=-h(t, \lambda) u_{\varepsilon}(t)$, we find that $u_{\varepsilon}(t, \lambda)$ satisfies the following integral equation:

$$
\begin{aligned}
u_{\varepsilon}(t, \lambda)= & x_{0}+\frac{\varepsilon}{b}\left[1-e^{-(b / \varepsilon) t}\right] x_{1} \\
& -\frac{1}{b} \int_{0}^{t} h(s, \lambda)\left[1-e^{-(b / \varepsilon)(t-s)}\right] u_{\varepsilon}(s, \lambda) d s .
\end{aligned}
$$

Similarly, for $u_{0}$ we obtain the integral equation

$$
u_{0}(t, \lambda)=x_{0}-\frac{1}{b} \int_{0}^{t} h(s, \lambda) u_{0}(s, \lambda) d s ;
$$

thus for the difference we have 


$$
\begin{aligned}
u_{\varepsilon}(t, \lambda)-u_{0}(t, \lambda)= & \frac{\varepsilon}{b}\left[1-e^{-(b / \delta) t}\right] x_{1} \\
& -\frac{1}{b} \int_{0}^{t} h(s, \lambda)\left[u_{\varepsilon}(s, \lambda)-u_{0}(s, \lambda)\right] d s \\
& +\frac{1}{b} \int_{0}^{t} h(s, \lambda) e^{-(b / s)(t-s)} u_{\varepsilon}(s, \lambda) d s .
\end{aligned}
$$

For convenience we define

$$
M_{T}(\lambda)=1+\frac{1}{b} e^{1 / b} \int_{0}^{T}|h(s, \lambda)| d s \int_{0}^{T}|h(s, \lambda)| d s .
$$

Observe that $M_{t}(\lambda) \leqq 2 \exp \left\{2 \int_{0}^{T}|h(s, \lambda)| d s\right\}$ since $\alpha e^{\alpha} \leqq e^{2 \alpha}$.

Lemma 1. For $t \in[0, T]$

$$
\begin{aligned}
& \left|p_{\varepsilon}(t, \lambda)\right| \leqq M_{T}(\lambda), \\
& \left|q_{\varepsilon}(t, \lambda)\right| \leqq \frac{\varepsilon}{b} M_{T}(\lambda), \\
& \left|p_{0}(t, \lambda)\right| \leqq M_{T}(\lambda) .
\end{aligned}
$$

Proof. From (5) we obtain

$$
\left|u_{\varepsilon}(t, \lambda)\right| \leqq\left|x_{0}\right|+\frac{\varepsilon}{b}\left|x_{1}\right|+\frac{1}{b} \int_{0}^{t}|h(s, \lambda)|\left|u_{\varepsilon}(s, \lambda)\right| d s
$$

Gronwall's lemma [3, p. 37] then implies that

$$
\left|u_{\varepsilon}(t, \lambda)\right| \leqq\left[\left|x_{0}\right|+\frac{\varepsilon}{b}\left|x_{1}\right|\right] M_{T}(\lambda)
$$

The first two statements of the lemma follow on setting $x_{0}=1, x_{1}=$ 0 and $x_{0}=0, x_{1}=1$, respectively. The last statement follows in the same manner from (6).

Theorem 1. For any $T>0$ and any fixed $\lambda, p_{s}(t, \lambda) \rightarrow p_{0}(t, \lambda)$ and $q_{s}(t, \lambda) \rightarrow 0$ as $\varepsilon \rightarrow 0+$, uniformly in $t \in[0, T]$.

Proof. That $q_{s}(t, \lambda) \rightarrow 0$ is obvious from Lemma 1 . Setting $x_{1}=$ $0, x_{0}=1$ in (7) yields

$$
\begin{aligned}
\mid p_{s}(t, \lambda) & -p_{0}(t, \lambda)\left|\leqq \frac{1}{b} \int_{0}^{t}\right| h(s, \lambda)|| p_{s}(s, \lambda)-p_{0}(s, \lambda) \mid d s \\
& +\frac{1}{b} M_{T}(\lambda) \int_{0}^{t}|h(s, \lambda)| e^{-\langle b / \varepsilon\rangle(t-s)} d s .
\end{aligned}
$$

For any $\delta>0$ we have 


$$
\begin{aligned}
\int_{0}^{t}|h(s, \lambda)| e^{-(b / s)(t-s)} d s & \leqq \int_{0}^{\max (t-\delta, 0)}|h(s, \lambda)| e^{-(b / s)(t-s)} d s+\int_{\max (t-\hat{\delta}, 0)}^{t}|h(s, \lambda)| d s \\
& \leqq e^{-(b / s) \hat{o}} \int_{0}^{T}|h(s, \lambda)| d s+\delta \sup _{0 \leqq t \leqq T}|h(t, \lambda)| \\
& \equiv \delta g(\lambda)+e^{-(b / s) \delta} k(\lambda) .
\end{aligned}
$$

Thus

$$
\begin{aligned}
\left|p_{s}(t, \lambda)-p_{0}(t, \lambda)\right| \leqq & \frac{1}{b} M_{T}(\lambda)\left[\delta g(\lambda)+e^{-(b / \varepsilon) \hat{o}} k(\lambda)\right] \\
& +\frac{1}{b} \int_{0}^{t}|h(s, \lambda)|\left|p_{\varepsilon}(s, \lambda)-p_{0}(s, \lambda)\right| d s
\end{aligned}
$$

application of Gronwall's lemma yields the inequality

$$
\left|p_{s}(t, \lambda)-p_{0}(t, \lambda)\right| \leqq \frac{1}{b}\left[\delta g(\lambda)+e^{-(b / s) \delta} k(\lambda)\right] M_{\lambda}(T)^{2} .
$$

Here the right-hand side can be made arbitrarily small by first choosing $\delta>0$ small and then requiring $\varepsilon$ to be sufficiently small.

Lemma 2. For $t \in[0, T]$,

$$
\begin{aligned}
& \left|p_{c}^{\prime}(t, \lambda)\right| \leqq \frac{2 b}{\varepsilon} e^{2 \int_{0}^{T}|h(s, \lambda)| d s},
\end{aligned}
$$

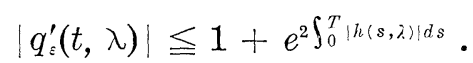

Proof. Differentiating (5), taking absolute values, and using the estimate (8), we get

$$
\left|u_{\varepsilon}^{\prime}(t, \lambda)\right| \leqq\left|x_{1}\right|+\frac{1}{\varepsilon}\left[\left|x_{0}\right|+\frac{\varepsilon}{b}\left|x_{1}\right|\right] M_{T}(\lambda) \int_{0}^{T}|h(s, \lambda)| d s ;
$$

setting in turn $x_{0}=1, x_{1}=0$ and $x_{0}=0, x_{1}=1$, and using the inequality $\alpha\left(1+\alpha e^{\alpha}\right) \leqq 2 e^{2 \alpha}$ for $\alpha>0$, yields the result.

Lemma 3. For $t \in[0, T]$,

$$
\begin{aligned}
& \left|p_{s}^{\prime \prime}(t, \lambda)\right| \leqq \frac{1}{\varepsilon^{2}}\left(2 b^{2}+\varepsilon \max _{[0, T]}|h(t, \lambda)|\right) e^{2 \int_{0}^{T}[h(s, \lambda) \mid d s}, \\
& \left|q_{s}^{\prime \prime}(t, \lambda)\right| \leqq\left(\frac{2 b}{\varepsilon}+\frac{2}{b} \max _{[0, T]}|h(t, \lambda)|\right) e^{2 \int_{0}^{T} \mid h(s, \lambda) i d s}, \\
& \left|p_{0}^{\prime}(t, \lambda)\right| \leqq \frac{2}{b}\left(\max _{[0, T]}|h(t, \lambda)|\right) e^{2 \int_{0}^{T}|h(s, \lambda)| d s} .
\end{aligned}
$$

Proof. The proof follows easily by using the differential equations (3), (4) themselves and the estimates contained in (9) and Lemma 1. 
THEOREM 2. For any $0<\tau<T, p_{\varepsilon}^{\prime}(t, \lambda) \rightarrow p_{0}^{\prime}(t, \lambda)$ uniformly for $t \in[0, T]$ and $q_{\varepsilon}^{\prime}(t, \lambda) \rightarrow 0$ uniformly for $t \in[\tau, T]$.

Proof. Differentiation of (5) and (6) yields the equations

$$
\begin{aligned}
& p_{\varepsilon}^{\prime}(t, \lambda)=-\frac{1}{\varepsilon} \int_{0}^{t} h(s, \lambda) e^{-(b / \varepsilon)(t-s)} p_{\varepsilon}(s, \lambda) d s, \\
& p_{0}^{\prime}(t, \lambda)=-\frac{1}{b} h(t, \lambda) p_{0}(t, \lambda), \\
& q_{\varepsilon}^{\prime}(t, \lambda)=e^{-(b / \varepsilon) t}-\frac{1}{\varepsilon} \int_{0}^{t} h(s, \lambda) e^{-(b / \varepsilon)(t-s)} q_{\varepsilon}(s, \lambda) d s .
\end{aligned}
$$

From the last of these equations we obtain, using Lemma 1,

$$
\left|q_{\varepsilon}^{\prime}(t, \lambda)\right| \leqq e^{-(b / \varepsilon) t}+\frac{1}{b} M_{T}(\lambda) \int_{0}^{t}|h(s, \lambda)| e^{-(b / \varepsilon)(t-s)} d s ;
$$

in the proof of Theorem 1 this integral was shown to approach zero, uniformly in $t \in[0, T]$, as $\varepsilon \rightarrow 0$. Since $e^{-(b / \varepsilon) t} \rightarrow 0$ uniformly for $t \in$ $[\tau, T]$, the second statement of theorem follows.

We turn to the first statement, writing for any fixed $\delta>0$

$$
\begin{aligned}
p_{\varepsilon}^{\prime}(t, \lambda)-p_{0}^{\prime}(t, \lambda)= & -\frac{1}{\varepsilon} \int_{0}^{\max (t-\delta, 0)} h(s, \lambda) e^{-(b / \varepsilon)(t-s)} p_{\varepsilon}(s, \lambda) d s \\
& -\frac{1}{\varepsilon} \int_{\max (t-\delta, 0)}^{t} h(s, \lambda) e^{-(b / \varepsilon)(t-s)} p_{\varepsilon}(s, \lambda) d s \\
& +\frac{1}{b} h(t, \lambda) p_{0}(t, \lambda) \equiv J_{1}+J_{2}+J_{3} .
\end{aligned}
$$

Since $\lambda e^{-\lambda} \leqq e^{-(1 / 2) \lambda}$, we have the estimate

$$
\begin{aligned}
\left|J_{1}\right| & \leqq \frac{1}{b \delta} \int_{0}^{\max (t-\delta, 0)} \frac{b}{\varepsilon}(t-s) e^{-(b / \varepsilon)(t-s)}|h(s, \lambda)|\left|p_{\varepsilon}(s, \lambda)\right| d s \\
& \leqq \frac{1}{b \delta} M_{T}(\lambda) e^{-(b / 2 \varepsilon) \delta} \int_{0}^{T}|h(s, \lambda)| d s
\end{aligned}
$$

where the final quantity tends to zero with $\varepsilon$. Also, since

$$
\int_{t-\delta}^{t} \frac{b}{\varepsilon} e^{-(b / s)(t-s)} d s=1-e^{-b \delta / \varepsilon},
$$

we have that

$$
\begin{aligned}
\left|b\left(J_{2}+J_{3}\right)\right| \leqq & \frac{b}{\varepsilon} \int_{t-\delta}^{t} e^{-(b / \varepsilon)(t-s)}|h(s, \lambda)|\left|p_{\varepsilon}(s, \lambda)-p_{0}(s, \lambda)\right| d s \\
& +e^{-b \delta / \varepsilon}|h(t, \lambda)|\left|p_{0}(t, \lambda)\right| .
\end{aligned}
$$


Setting $\mu_{\varepsilon}(\lambda, T)=\sup _{t \in[0, T]}\left|p_{\varepsilon}(t, \lambda)-p_{0}(t, \lambda)\right|, \nu(\lambda, T)=\sup _{t \in[0, T]}|h(t, \lambda)|$, we get that

$$
\begin{aligned}
\left|b\left(J_{2}+J_{3}\right)\right| \leqq & e^{-b \delta / \varepsilon} \nu(\lambda, T) \sup _{t \in[0, T]} p_{0}(t, \lambda) \mid \\
& +\mu_{\varepsilon}(\lambda, T) \nu(\lambda, T)\left(1-e^{-b \delta / \varepsilon}\right) ;
\end{aligned}
$$

the right-hand side tends to zero with $\varepsilon$ by Theorem 1 .

2. The problem in a Hilbert space. Let $H$ be a Hilbert space, $E_{\lambda}$ the resolution of the identity for the self-adjoint operator $A$, and define operators $P_{\varepsilon}, P_{0}, Q_{\varepsilon}$ on $H$ by

$$
\begin{aligned}
& P_{\varepsilon}(t)=\int_{-\infty}^{\infty} p_{\varepsilon}(t, \lambda) d E_{\lambda}, \\
& P_{0}(t)=\int_{-\infty}^{\infty} p_{0}(t, \lambda) d E_{\lambda}, \\
& Q_{\varepsilon}(t)=\int_{-\infty}^{\infty} q_{\varepsilon}(t, \lambda) d E_{\lambda} ;
\end{aligned}
$$

let $T>0$ be a fixed number. Let $D$ denote the (dense) domain of the operator

$$
\left(I+A+\max _{[0, T]}|h(t, A)|\right) \exp \left\{2 \int_{0}^{T}|h(s, A)| d s\right\}
$$

defined as

$$
\int_{-\infty}^{\infty}\left(1+\lambda+\max _{[0, T]}|h(t, \lambda)|\right) e^{2} \int_{0}^{T}|h(s, \lambda)| d s d E_{\lambda}
$$

then

$$
D=\left\{x \in H: \int_{-\infty}^{\infty}\left(1+\lambda+\max _{[0, T]}|h(t, \lambda)|\right)^{2} e^{4} \int_{0}^{T}|h(s, \lambda)| d s d\left(E_{\lambda} x, x\right)<\infty\right\} .
$$

$D$ is contained in the domains of $P_{\varepsilon}, P_{0}, Q_{\varepsilon}$, because for $x \in D$

$$
\begin{aligned}
& \left\|P_{\varepsilon}(t) x\right\|^{2}=\int_{-\infty}^{\infty}\left|p_{\varepsilon}(t, \lambda)\right|^{2} d\left(E_{\lambda} x, x\right) \leqq \int_{-\infty}^{\infty}\left[M_{T}(\lambda)\right]^{2} d\left(E_{\lambda} x, x\right)
\end{aligned}
$$

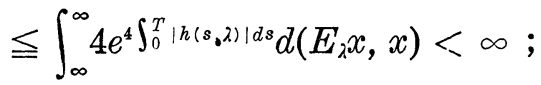

similar calculations are valid for $P_{0}, Q_{0}$. Also, if $x \in D$, then $P_{\varepsilon} x, P_{0} x$, $Q_{\varepsilon} x \in D(A)$, as is shown by calculations like the following for $P_{\varepsilon} x$ :

$$
\begin{aligned}
& \int_{-\infty}^{\infty} \lambda^{2} d\left\|E_{\lambda} P_{\varepsilon} x\right\|^{2}=\int_{-\infty}^{\infty} \lambda^{2} d\left\|E_{\lambda} \int_{-\infty}^{\infty} p_{\varepsilon}(t, \mu) d E_{\mu} x\right\|^{2} \\
& =\int_{-\infty}^{\infty} \lambda^{2} d\left\|\int_{-\infty}^{\lambda} p_{\varepsilon}(t, \mu) d E_{\mu} x\right\|^{2} \leqq \int_{-\infty}^{\infty} \lambda^{2} d \int_{-\infty}^{\lambda} 4 e^{4} \int_{0}^{T}|h(s, \mu)| d s d\left\|E_{\mu} x\right\|^{2} \\
& =4 \int_{-\infty}^{\infty} \lambda^{2} e^{4} \int_{0}^{T}|h(s, \lambda)| d s d\left\|E_{\lambda} x\right\|^{2}<\infty \text {. }
\end{aligned}
$$


Defining $U_{\varepsilon}(t)=P_{\varepsilon}(t) x_{0}+Q_{\varepsilon}(t) x_{1}$ for $\varepsilon>0$ and $U_{0}(t)=P_{0}(t) x_{0}$, we have

Lemma 4. $U_{\varepsilon}(t), U_{0}(t)$ solve the problems (1), (2), respectively, on $[0, T]$.

Proof. We shall prove only the statement concerning $U_{\varepsilon}$; that $U_{0}$ solves (2) is proved similarly. In view of the fact that $p_{\varepsilon}(t, \lambda)$ and $q_{\varepsilon}(t, \lambda)$ satisfy the differential equation (3), it is enough to show that the first and second derivatives of $P_{\varepsilon}, Q_{\varepsilon}$ can be taken under the integral sign; that is, for $x \in D$,

$$
\begin{aligned}
\left(\frac{d}{d t}\right)^{\nu} P_{\varepsilon}(t) x= & \int_{-\infty}^{\infty}\left(\frac{d}{d t}\right)^{\nu} p_{\varepsilon}(t, \lambda) d E_{2} x, \\
& \left(\frac{d}{d t}\right)^{\nu} Q_{\varepsilon}(t) x \sim=\int_{-\infty}^{\infty}\left(\frac{d}{d t}\right)^{\nu} q_{\varepsilon}(t, \lambda) d E_{\lambda} x \quad(\nu=1,2) ;
\end{aligned}
$$

we present a proof of the statement for $P_{\varepsilon}$. By the mean value theorem we have that

$$
\frac{1}{h}\left[P_{\varepsilon}(t+h) x-P_{\varepsilon}(t) x\right]=\int_{-\infty}^{\infty} \frac{d}{d t} p_{\varepsilon}\left(t^{\prime}, \lambda\right) d E_{\lambda} x
$$

for some $t^{\prime}$ between $t$ and $t+h$. Now

$$
\begin{aligned}
& \left\|\int_{-\infty}^{\infty} \frac{d}{d t} p_{s}\left(t^{\prime}, \lambda\right) d E_{\lambda} x-\int_{-\infty}^{\infty} \frac{d}{d t} p_{\varepsilon}(t, \lambda) d E_{\lambda} x\right\|^{2} \\
& \leqq \int_{-\infty}^{\infty}\left|\frac{d}{d t} p_{\varepsilon}\left(t^{\prime}, \lambda\right)-\frac{d}{d t} p_{\varepsilon}(t, \lambda)\right|^{2} d\left\|E_{\lambda} x\right\|^{2},
\end{aligned}
$$

so the desired result is a consequence of the Lebesgue dominated convergence theorem if we show that the integrand of the last integral is bounded by a function integrable with respect to the measure $\left\|E_{i} x\right\|^{2}$. To this end restrict $h$ to be small enough that $0 \leqq t+h \leqq$ $T$. Then from Lemma 2

$$
\left|\frac{d}{d t} p_{\varepsilon}\left(t^{\prime}, \lambda\right)\right|^{2},\left.\frac{d}{d t} p_{\varepsilon}(t, \lambda)\right|^{2} \leqq \frac{4 b^{2}}{\varepsilon^{2}} e^{4} \int_{0}^{T}|h(s, \lambda)| d s,
$$

which is integrable for each fixed $\varepsilon>0$. The proof of the case $\nu=$ 2 is similar but uses Lemma 3 instead of Lemma 2 .

LEMma 5. The solutions of the problems (1), (2) are unique.

Proof. We shall show that $P_{\varepsilon}(t) x_{0}$ is the only solution of (1) with $x_{1}=0$; the omitted cases are similar. Let $h_{n}(t, A)$ for any integer $n$ be the bounded operator 


$$
h_{n}(t, A) \equiv \int_{-n}^{n} h(t, \lambda) d E_{\lambda}=\left(E_{n}-E_{-n}\right) h(t, A) .
$$

Suppose $R_{\varepsilon}(t)$ is a solution of (1) with $x_{1}=0$, and set $z_{\varepsilon}(t)=P_{\varepsilon}(t) x_{0}$ $R_{\varepsilon}(t)$. Set $z_{\varepsilon, n}(t)=\left(E_{n}-E_{-n}\right) z_{\varepsilon}(t)$; then

$$
\begin{aligned}
& \varepsilon z_{\varepsilon, n}^{\prime \prime}+b z_{\varepsilon, n}^{\prime}+h_{n}(t, A) z_{\varepsilon, n} \\
& \quad=\left(E_{n}-E_{-n}\right)\left(\varepsilon z_{\varepsilon}^{\prime \prime}+b z_{\varepsilon}^{\prime}+h(t, A) z_{\varepsilon}\right)=0,
\end{aligned}
$$

and $z_{\varepsilon, n}(0)=0, z_{\varepsilon, n}^{\prime}(0)=0$. If we show that $z_{\varepsilon, n} \equiv 0$, we will have $0=\lim _{n \rightarrow \infty} z_{\varepsilon, n}(t)=z_{\varepsilon}(t)$, as desired. Now $z_{\varepsilon, n}$ satisfies the integral equation

$$
z_{\varepsilon, n}(t)=-\frac{1}{b} \int_{0}^{t}\left[1-e^{-(b / \varepsilon)(t-s)}\right] h_{n}(s, A) z_{\varepsilon, n}(s) d s,
$$

whence

$$
\left\|z_{\varepsilon, n}(t)\right\| \leqq \frac{1}{b} \int_{0}^{t}\left\|h_{n}(s, A)\right\|\left\|z_{\varepsilon, n}(s)\right\| d s,
$$

and $z_{\varepsilon, n} \equiv 0$ follows in standard fashion.

THeOREM 3. For $x_{0}, x_{1} \in D$ we have

$$
\lim _{\varepsilon \rightarrow 0+}\left\|U_{\varepsilon}(t)-U_{0}(t)\right\|=0
$$

uniformly for $t \in[0, t]$, and for any $\tau>0, \tau<T$,

$$
\lim _{\varepsilon \rightarrow 0+}\left\|U_{\varepsilon}^{\prime}(t)-U_{0}^{\prime}(t)\right\|=0
$$

uniformly for $t \in[\tau, T]$.

Proof. It is necessary to show that

$$
\begin{aligned}
& \lim _{\varepsilon \rightarrow 0+} \int_{-\infty}^{\infty}\left|p_{\varepsilon}(t, \lambda)-p_{0}(t, \lambda)\right|^{2} d\left\|E_{2} x_{0}\right\|^{2}=0, \\
& \lim _{\varepsilon \rightarrow 0+} \int_{-\infty}^{\infty}\left|q_{\varepsilon}(t, \lambda)\right|^{2} d\left\|E_{\lambda} x_{1}\right\|^{2}=0, \\
& \lim _{\varepsilon \rightarrow 0+} \int_{-\infty}^{\infty}\left|p_{\varepsilon}^{\prime}(t, \lambda)-p_{0}^{\prime}(t, \lambda)\right|^{2} d\left\|E_{\lambda} x_{0}\right\|^{2}=0, \\
& \lim _{\varepsilon \rightarrow 0+} \int_{-\infty}^{\infty}\left|q_{\varepsilon}^{\prime}(t, \lambda)\right|^{2} d\left\|E_{\lambda} x_{1}\right\|^{2}=0 .
\end{aligned}
$$

Since, by Lemma 1 ,

$$
\left|p_{\varepsilon}(t, \lambda)-p_{0}(t, \lambda)\right|^{2} \leqq 2\left|p_{\varepsilon}(t, \lambda)\right|^{2}+2\left|p_{0}(t, \lambda)\right|^{2} \leqq 4 M_{T}(\lambda),
$$

the first result follows from Theorem 1 and the Lebesgue dominated convergence theorem. The remaining statements follow in a similar manner. 


\section{REFERENCES}

1. L. Bobisud and J. Calvert, Singularly perturbed differential equations in a Hilbert space, J. Math. Anal. Appl., 30 (1970), 113-127.

2. L. Bobisud and R. Hersh, Perturbation and approximation theory for higher-order abstract Cauchy problems, Rocky Mountain J. Math., 2 (1972), 57-73.

3. E. Coddington and N. Levinson, Theory of Ordinary Differential Equations, McGraw-Hill, 1955.

4. A. Friedman, Singular perturbations for the Cauchy problem and for boundary value problems, J. Differential Equations, 5 (1969), 226-261.

5. J. Kisyński, Sur les équations hyperboliques avec petit paramètre, Colloq. Math., 10 (1963), 331-343.

6. B. Latil, Singular perturbations of Cauchy's problem, J. Math. Anal. Appl., 23 (1968), 683-698.

7. H. Nur, Singular perturbations of differential equations in abstract spaces, Pacific

J. Math., 36 (1971), 775-780.

8. A. Schoene, Semigroups and a class of singular perturbation problems, Indiana Univ. Math. J., 20 (1970), 247-263.

9. J. Smoller, Singular perturbations and a theorem of Kisyński, J. Math. Anal. Appl., 12 (1965), 105-114.

10. Singular perturbations of Cauchy's problem, Comm. Pure Appl. Math., 18 (1965), 665-677.

11. M. Sova, Équations hyperboliques avec petit paramètre dans les espaces de Banach généraux, Colloq. Math., 21 (1970), 303-320.

Received April 2, 1973.

UNIVERSITY OF IDAHO 



\section{PACIFIC JOURNAL OF MATHEMATICS}

\section{EDITORS}

RICHARD ARENS (Managing Editor)

University of California

Los Angeles, California 90024

R. A. Beaumont

University of Washington

Seattle, Washington 98105
J. DugundJI

Department of Mathematics University of Southern California Los Angeles, California 90007

D. Gilbarg AND J. Milgram Stanford University

Stanford, California 94305

\section{ASSOCIATE EDITORS}
E. F. BECKENBACH
B. H. NeUmanN
F. WOLF
K. YOSHIDA

\section{SUPPORTING INSTITUTIONS}

UNIVERSITY OF BRITISH COLUMBIA CALIFORNIA INSTITUTE OF TECHNOLOGY

UNIVERSITY OF CALIFORNIA

MONTANA STATE UNIVERSITY

UNIVERSITY OF NEVADA

NEW MEXICO STATE UNIVERSITY

OREGON STATE UNIVERSITY

UNIVERSITY OF OREGON

OSAKA UNIVERSITY
UNIVERSITY OF SOUTHERN CALIFOF

STANFORD UNIVERSITY

UNIVERSITY OF TOKYO

UNIVERSITY OF UTAH

WASHINGTON STATE UNIVERSITY UNIVERSITY OF WASHINGTON

AMERICAN MATHEMATICAL SOCIET NAVAL WEAPONS CENTER 


\section{Pacific Journal of Mathematics}

\section{Vol. 54, No. 1 \\ May, 1974}

Ralph K Amayo, Engel Lie rings with chain conditions ..................

Bernd Anger and Jörn Lembcke, Hahn-Banach type theorems for hypolinear

functionals on preordered topological vector spaces ..................

Gregory Frank Bachelis and Samuel Ebenstein, On $\Lambda(p)$ sets ................

Harvey Isaac Blau, Indecomposable modules for direct products of finite

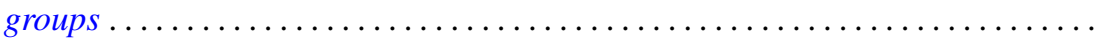

Larry Eugene Bobisud and James Calvert, Singular perturbation of a

time-dependent Cauchy problem in a Hilbert space ................

Walter D. Burgess and Robert Raphael, Abian's order relation and orthogonal

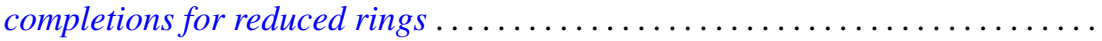

James Diederich, Representation of superharmonic functions mean continuous at

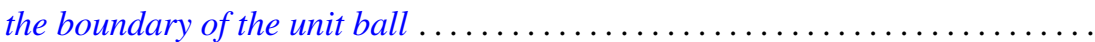

Aad Dijksma and Hendrik S. V. de Snoo, Self-adjoint extensions of symmetric

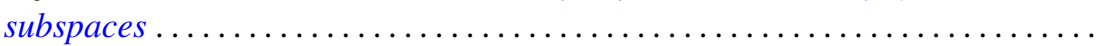

Gustave Adam Efroymson, A Nullstellensatz for Nash rings . . . . . . . . . . . . .

John D. Elwin and Donald R. Short, Branched immersions onto compact orientable surfaces . . . . . . . . . . . . . . . . . . . . . . . . .

John Douglas Faires, Comparison of the states of closed linear

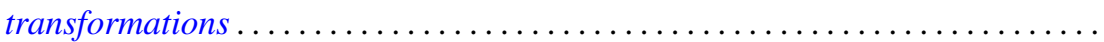

Joe Wayne Fisher and Robert L. Snider, On the von Neumann regularity of rings with regular prime factor rings .

Franklin Takashi Iha, A unified approach to boundary value problems on compact intervals

Palaniappan L. Kannappan and Che Tat $\mathrm{Ng}$, On functional equations connected with directed divergence, inaccuracy and generalized directed divergence

Samir A. Khabbaz and Elias Hanna Toubassi, The module structure of Ext $(F, T)$ over the endomorphism ring of $T$...

Garo K. Kiremidjian, On deformations of complex compact manifolds with boundary.

Dimitri Koutroufiotis, Mappings by parallel normals preserving principal

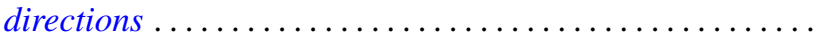

W. K. Nicholson, Semiperfect rings with abelian adjoint group

Norman R. Reilly, Extension of congruences and homomorphisms to translational hulls

Sadahiro Saeki, Symmetric maximal ideals in $M(G)$

Brian Kirkwood Schmidt, On the homotopy invariance of certain functors ...

H. J. Shyr and T. M. Viswanathan, On the radicals of lattice-ordered rings ...

Indranand Sinha, Certain representations of infinite group algebras ...

David Smallen, The group of self-equivalences of certain complexes ...

Kalathoor Varadarajan, On a certain problem of realization in homotopy

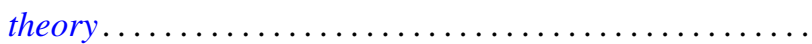

